\title{
Remarks on the skull morphology of Canis lupaster Hemprich and Herenberg, 1832 from the collection of the Natural History Museum “G. Doria” of Genoa, Italy
}

\author{
Davide Federico Bertè
}

\begin{abstract}
Canis lupaster is a canid that lives in North Africa. In the past, this species was considered a subspecies of golden jackal (Canis aureus), a subspecies of wolf (Canis lupus), or a separated species. Since 2011 genetic data have demonstrated that $C$. lupaster is not a golden jackal and that it is more closely related to wolf lineage. The recent interest on $C$. lupaster lead to the publication of some papers on this topic, but the vast majority concerned genetic data. In this paper a morphological approach is presented. Twelve specimens, collected in Libya between 1926 and 1931 and now stored in the Natural History Museum of Genoa, are described here. C. lupaster is compared with the African golden jackal and with the wolf. MANOVA, PCA and discriminant analysis were performed. C. lupaster show many differences and is well separated both from wolf and from African golden jackal. Measures and ratios, that allow to rapidly recognise among these species, were identified. These ratios could be a useful tool for field researchers to quickly identify the correct species.
\end{abstract}

Key words: Canis lupaster, Canis anthus, African golden jackal, wolf, skull morphology, Libya.

Riassunto - Osservazioni sulla morfologia del cranio di Canis lupaster Hemprich and Herenberg 1832 dalla collezione del Museo di Storia Naturale "G. Doria" di Genova, Italia.

Canis lupaster è un canide che vive in Nord Africa. In passato questa specie è stata considerata come una sottospecie dello sciacallo dorato (Canis aureus), come sottospecie del lupo (Canis lupus), o come specie separata. Dal 2011 dati genetici hanno dimostrato che C. lupaster non è uno sciacallo dorato e che è più affine alla genealogia del lupo. Il recente interesse su $C$. lupaster ha provocato la pubblicazione di alcuni articoli sull'argomento, tutti di taglio genetico. In questo lavoro viene presentato un approccio morfologico. Dodici esemplari, catturati in Libia tra il 1926 e il 1931 e ora conservati presso il Museo di Storia Naturale di Genova, sono qui descritti. C. lupaster è stato comparato con lo sciacallo dorato africano e con il lupo. Sono state eseguite MANOVA, PCA e analisi discriminante. C. lupaster mostra molte differenze ed è ben distinto sia dal lupo che dallo sciacallo dorato africano. Sono state individuate misure e rapporti che permettono di distinguere tra queste tre specie. Questi rapporti potrebbero essere utili per i ricercatori sul campo per identificare rapidamente la specie corretta.

Associazione Culturale 3P (Progetto Preistoria Piemonte), Via lunga 38, San Mauro Torinese, 10099 Torino, Italia.

E-mail: davide.berte@gmail.com

(C) 2017 Davide Federico Bertè

Received: 25 October 2016

Accepted for publication: 27 December 2016
Parole chiave: Canis lupaster, Canis anthus, sciacallo dorato africano, lupo, morfologia cranica, Libia.

\section{INTRODUCTION}

Canis lupaster Hemprich and Herenberg 1832 (Fig. 1) is a canid that lives in North Africa (Gaubert et al., 2012). The taxonomic position of $C$. lupaster has changed many times in the last centuries. Initially $C$. lupaster was described as true species by Hemprich and Herenberg (1832), and this position was shared by other authors (Beaux, 1927; Zammarano, 1930; Flower, 1932). After that C. lupaster was considered as a subspecies of Canis aureus because the distribution and the body-size is closer to C. aureus (Anderson \& Winton, 1902; Schwarz, 1926a, 1926b; Ellerman \& Morrison-Scott, 1951; Setzer, 1961). Despite this, some authors considered this species as separated from $C$. aureus on the basis of ethological observations (Flower, 1932; Hoogstraal, 1964; Hufnagl, 1972).

In 1831 Sykes discovered the Indian wolf Canis lupus pallipes and the similarity with $C$. lupaster was noted in the following years (Anderson, 1902). Ferguson (1981) suggests that $C$. lupaster should be considered as subspecies of $C$. lupus from a morphological point of view. Measurements of the skull length, mandible and carnassial of $C$. lupaster overlap the lower limits of $C$. lupus arabs and show a distinct gap with those of C. aureus (Ferguson, 1981), and according to the Bergmann's rule, $C$. lupaster is probably a small wolf rather than a giant jackal (Ferguson, 1981).

Genetic analyses have revealed that $C$. lupaster is not a golden jackal (Rueness et al., 2011) and it is more similar to the Canis lupus lineage. Gaubert et al. (2012) found four distinct lineages of wolf: C. lupus/familiaris (Holarctic wolves and $\operatorname{dogs}$ ), C. l. chanco (Himalayan wolf), C. l. pallipes (Indian wolf) and C. l. lupaster (African wolf). The lineage of $C$. l. lupaster is relatively ancient, with a time to most recent common ancestor estimated at $288 \mathrm{k}$ years ago (Gaubert et al., 2012). However, despite phenotypical and ethological differences, $C$. lupaster mtDNA was detected in African C. aureus, suggesting a hybridization (Gaubert et al., 2012). At the moment $C$. lupaster is considered a separated species thanks to genetic on mitochondrial and genomic DNA (Koepfli et al. 2015; Rueness et al., 2015; Urios et al., 2015), phenotypic (Gaubert et al., 2012) and morphologic data (Spassov \& Stoyanov, 2014). 


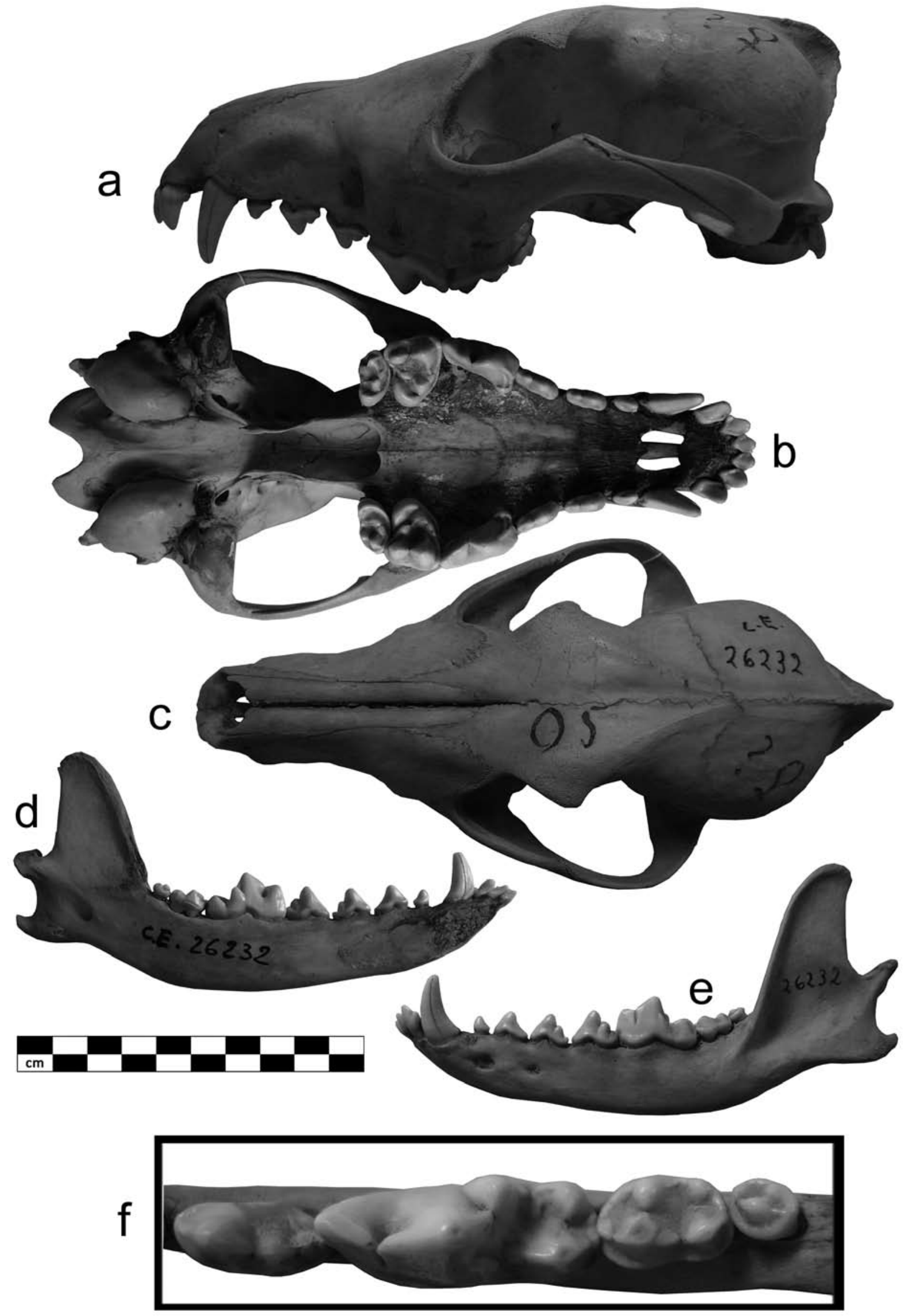

Fig. 1 - Specimen MSNG 26232. a) skull in left lateral view; b) skull in ventral view; c) skull in dorsal view; d) left hemimandible in lingual view; e) left hemimandible in labial view; f) occlusal view of P4-M3. 
Koepfli et al. (2015) separate European Canis aureus from African Canis aureus; this latter, following Cuvier, is called Canis anthus. Cuvier used the name C. anthus to describe an African golden jackal from Senegal as different from Eurasian golden jackal. In the opinion of Koepfli et al. (2015) the name C. aureus could be referred only to Eurasian specimen while the African specimens must be considered as $C$. anthus. In the paper of Koeplki et al. (2015) an exhaustive analysis on different populations of African golden jackal however is absent. In the work of Van Valkenburgh \& Wayne (1994) the specimens from different populations of "African golden jackal" are considered all together but the authors recognise that the population of North Africa ( C. lupaster) is quite different. In the supplementary material Koepfli et al. (2015) cite the work of Rueness et al. (2015) but a comparison is made only between Eurasian golden jackal and African wolf (C. lupaster), while comparison between African golden jackal (C. anthus) and African wolf (C. lupaster) is lacking. Gaubert et al. (2012) compared $C$. lupaster with East African golden jackal and they found significant differences; Gaubert et al. (2012) also reported some differences between Eurasian and African jackals, although they didn't separate the two species.

In this work I follow Koepfli et al. (2015) considering the "African golden jackal" as $C$. anthus but I suggest that $C$. lupaster must be considered as a different taxonomic unit; this opinion is based on genetic evidence reported in literature (Rueness et al. 2011; Gaubert et al. 2012) and on morphological differences reported below in this paper.

The taxonomic status of $C$. lupaster is important to establish adequate conservation measures on wild populations. While papers on genetic data are increasing, no recent papers on skeletal morphology are available. Aim of this paper is to identify new morphological and morphometric features that allow to easily distinguish C. lupaster both from C. lupus and "African golden jackal". On a morphological point of view some typical features of C. lupaster are expected to be find because the separation from wolf lineage is relatively ancient and, on counterpart, some convergence with $C$. anthus due to environmental conditions are expected to be find.

\section{MATERIALS AND METHODS}

The specimens of $C$. lupaster in exam are stored in Natural History Museum "G. Doria" of Genoa (MSNG). In the collection are present 12 skulls; male and female are equally represented. Ten of the specimens are collected in Cyrenaica (Libya) between 1926 and 1931 and two are from captivity. The specimens MSNG 26228, MSNG 26229, MSNG 26230, MSNG 26231, MSNG 26232, MSNG 26233, MSNG 26449 are collected at oasis of Giarabub in $1926-1927$ by C. Confalonieri. The specimens MSNG 31630, MSNG 31632, MSNG 31635 are collected at Es Sahabi, oasis of Cufra, in 1931 by marquis Patrizi. The specimen MSNG 32184 was a gift to Circo Mannucci by Captain Vassallo in 1933. The specimen MSNG 34260 was a gift of B. and S. Sonnenberger to the Zoo of Nervi
(Genoa), where has lived between 1932 and 1937. The skins of nine of these specimens are stored in the Natural History Museum "G. Doria" of Genoa.

A comparison of the skull of $C$. lupaster with African golden jackals and European wolf is here presented.

Data on recent $C$. lupus from the Apennine area were taken from Bertè (2013) and include 115 individuals (52 males and 63 females) belonging to the Italian subspecies C. lupus italicus (Nowak \& Federoff, 2002; Boitani et al., 2003). The specimens considered here are stored at ISPRA, Department of Zoology of Sapienza, University of Rome (DZR), the Natural History Museum "G. Doria" of Genoa (MSNG), the Natural History Museum of Milan (MSNM) and the Regional Natural History Museum of Turin (MRSNT).

The "African golden jackal" specimens are stored at the Natural History Museum "G. Doria" of Genoa and Natural History Museum of Milan. These specimens were collected in African localities (5 from Eritrea, 4 from Somalia, 1 from Tunisia, 1 from Algeria, 1 from Libya) and, following Koepfli et al. (2015), now they must be considered as $C$. anthus.

Morphometric data were taken with a standard calliper. The measures, taken following Von den Driesch (1976), are length and breadth of each tooth, the greatest breadth of $\mathrm{P}^{4}$, the talonid length of $\mathrm{M}_{1}$, and those listed and described in Tab. 1. The measures are reported in Tabs. 2 and 3.

All the statistical analyses are performed with the software PAST version 2.08 (Hammer et al., 2001). A Principal Component Analysis (PCA) was performed to visualise variance in skull and mandible measures across samples using Principal Component vectors. Skull and related mandible were analysed together. A Multivariate Analysis of Variance (MANOVA) was also performed. Wilk's lambda value is reported. If the MANOVA shows significant overall difference between groups, the analysis can proceed by pairwise comparisons. Bonferroni correction for multiple test is applied and $\mathrm{P}$ values (multiplied by the number of pairwise comparisons) are reported. ANOVA is performed only on PC1. If ANOVA shows significant difference of the means (low P), a "post-hoc" pairwise comparisons is used, based on Tukey's HSD (Honestly Significant Difference) test. Sample sizes do not have to be equal for the version of Tukey's test used. A discriminant analysis was also performed on the data to confirming or rejecting the hypothesis that two species are morphologically distinct. A confusion matrix was produced; it is a table with the true class in rows and the predicted class in columns. The diagonal elements represent correctly classified combinations, while the cross-diagonal elements represent misclassified combinations. On this data the producer accuracy (PA) was calculated as the percentage of correctly classified values in a given class on the total number of values in that class, and the user accuracy (UA) as the percentage of correctly classified values in a given class divided by the number of values classified for that class; the overall accuracy (OA) was calculated as an average value following the formula: (true positives + true negatives)/(true positives + true negatives + false positives + false negatives). 
Tab. 1 - List and description of the measures taken on the skull and mandible.

\begin{tabular}{|c|c|}
\hline Abbreviation & Description \\
\hline TL & Total length - akrokranion-prosthion \\
\hline CL & Condylobasal length - aboral border of the occipital condiles-prosthion \\
\hline $\mathrm{BL}$ & Basal length - basal-prosthion \\
\hline UNL & Upper neurocranium length - akrokranion-frontal midpoint \\
\hline VcL & Viscerocranium length - nasion-prosthion \\
\hline FL & Facial length - frontal midpoint-prosthion \\
\hline GLN & Greatest length of the nasals - nasion-rhinion \\
\hline SL & Snout length - oral border of the orbits-prosthion \\
\hline MPL & Median palatal length - staphylion-prosthion \\
\hline PL & Palatal length - median point intersection choanae-prosthion \\
\hline LhP & Length of the horizontal part of the palatine - staphylion-palatinoorale \\
\hline LCR & Length of the cheektooth row - measured along the alveoli on the buccal side \\
\hline LMR & Length of the molar row \\
\hline LPR & Length of the premolar row \\
\hline GdAB & Greatest diameter auditory bulla \\
\hline Gmb & Greatest mastoid breadth - otion-otion \\
\hline BdeAM & Breadth dorsal to the external auditory meatus \\
\hline GBOC & Greatest breadth occipital condili \\
\hline GbbPp & Greatest breadth of the bases of the paraoccipital processes \\
\hline GBFM & Greatest breadth of the foramen magnum \\
\hline HFM & Height of the foramen magnum - basion-opisthion \\
\hline GNB & Greatest neurocranium breadth - euryon-euryon \\
\hline ZB & Zygomatic breadth - zygion-zygion \\
\hline LBS & Least breadth of skull - breadth at postorbital constrinction \\
\hline FB & Frontal breadth - ectorbitale-ectorbitale \\
\hline LbbO & Least breadth between the orbits - entorbitale-entorbitale \\
\hline GPB & Greatest palatal breadth - across outer borders of the alveoli \\
\hline LPB & Least palatal breadth - behind the canines \\
\hline $\mathrm{BCA}$ & Breadth at the canine alveoli \\
\hline $\mathrm{GiHO}$ & Greatest inner height of the orbit \\
\hline SH & Skull height \\
\hline Hot & Height of the occipital triangle - akrokranion-basion \\
\hline HTO & Height from toothrow to orbit \\
\hline DJ & Depth of jugal \\
\hline Tlm & Total length - condyle process-infradentale \\
\hline Lapi & Length: angular process-infradentale \\
\hline Lii & Length: indentation between condyle process and angular process- infradentale \\
\hline Lcpc & Length: condyle process-aboral border canine alveoli \\
\hline Lic & Length: indentation between condyle process and angular process-aboral border canine alveolus \\
\hline Lapc & Length angular process-aboral border canine alveolus \\
\hline Lmr & Length of the molar row \\
\hline L C-M3 & Length from canine to M3 \\
\hline L P1-M3 & Length from P1 to M3 \\
\hline L P1-M2 & Length from $\mathrm{P} 1$ to $\mathrm{M} 2$ \\
\hline L P2-M3 & Length from $\mathrm{P} 2$ to $\mathrm{M} 3$ \\
\hline L P1-P4 & Length from P1 to P4 \\
\hline L P2-P4 & Length from P2 to P4 \\
\hline La M1 & Length of the carnassial alveolus \\
\hline Hm P1 & Heigth of the mandible behind P1 \\
\hline $\mathrm{Hm}$ P2P3 & Heigth of the mandible between P2 and P3 \\
\hline Hm M1 & Heigth of the mandible behind M1 \\
\hline GT M1 & Greatest thickness of the body of jaw below M1 \\
\hline HVR & Heigth of the vertical ramus - basal point angular process-coronion \\
\hline
\end{tabular}


Tab. 2 - Measures taken on skull and mandible.

\begin{tabular}{|c|c|c|c|c|c|c|c|c|c|c|c|c|}
\hline $\mathbf{N}$ & $\begin{array}{r}\text { MSNG } \\
26231\end{array}$ & $\begin{array}{r}\text { MSNG } \\
26232\end{array}$ & $\begin{array}{r}\text { MSNG } \\
26233\end{array}$ & $\begin{array}{r}\text { MSNG } \\
31632\end{array}$ & $\begin{array}{r}\text { MSNG } \\
31635\end{array}$ & $\begin{array}{r}\text { MSNG } \\
34260\end{array}$ & $\begin{array}{r}\text { MSNG } \\
26228\end{array}$ & $\begin{array}{r}\mathrm{MSNG} \\
26229\end{array}$ & $\begin{array}{r}\text { MSNG } \\
26230\end{array}$ & $\begin{array}{r}\text { MSNG } \\
26449\end{array}$ & $\begin{array}{r}\text { MSNG } \\
31630\end{array}$ & $\begin{array}{r}\text { MSNG } \\
32184 \\
\end{array}$ \\
\hline Sex & $\mathrm{F}$ & $\mathrm{F}$ & $\mathrm{F}$ & $\mathrm{F}$ & $\mathrm{F}$ & $\mathrm{F}$ & $M$ & $\mathrm{M}$ & $\mathrm{M}$ & $\mathrm{M}$ & $M$ & $\mathrm{M}$ \\
\hline TL & 158.0 & 170.1 & 167.0 & 171.0 & 167.5 & 155.3 & 183.0 & 154.6 & 173.0 & 178.0 & 171.0 & 180.5 \\
\hline CL & 146.8 & 154.3 & 155.0 & & 152.0 & 146.0 & 155.3 & 144.7 & 155.4 & 158.0 & & 162.0 \\
\hline BL & 139.5 & 146.6 & 147.4 & & 145.0 & 138.7 & 171.6 & 136.7 & 149.0 & 149.0 & 141.6 & 153.0 \\
\hline UNL & 76.0 & 75.6 & 76.0 & 74.0 & 70.4 & 75.0 & 83.0 & 76.2 & 78.5 & 81.0 & 74.0 & 79.5 \\
\hline VcL & 69.0 & 74.3 & 69.0 & 77.4 & 77.0 & 69.0 & 78.0 & 72.3 & 73.3 & 77.0 & 79.0 & 81.0 \\
\hline FL & 89.0 & 93.5 & 91.0 & 97.4 & 97.0 & 92.0 & 100.0 & 84.6 & 94.7 & 97.0 & 97.0 & 101.0 \\
\hline GLN & 50.0 & 56.3 & & 57.4 & 56.0 & 50.8 & 56.3 & 55.3 & 51.2 & 58.2 & 60.0 & 59.5 \\
\hline SL & 64.5 & 68.6 & 67.9 & 70.0 & 70.4 & 67.4 & 74.0 & 62.5 & 70.4 & 71.3 & 69.6 & 73.7 \\
\hline MPL & 76.6 & 80.6 & 79.0 & 81.2 & 79.6 & 76.0 & 84.0 & 76.6 & 79.7 & 82.0 & 77.3 & 87.2 \\
\hline PL & 74.8 & 80.0 & 77.0 & 80.7 & 78.7 & 75.0 & 83.6 & 75.5 & 78.6 & 80.7 & 77.0 & 85.5 \\
\hline LhP & 27.0 & 29.0 & 29.6 & 28.0 & 26.0 & 25.0 & 29.2 & 28.0 & 28.7 & 27.7 & 27.3 & 29.7 \\
\hline LCR & 56.0 & 60.0 & 58.0 & 59.8 & 59.3 & 55.8 & 65.0 & 54.5 & 59.0 & 59.8 & 58.6 & 61.0 \\
\hline LMR & 16.3 & 17.7 & 16.8 & 17.0 & 17.2 & 17.4 & 18.5 & 18.0 & 16.0 & 17.0 & 16.8 & 18.7 \\
\hline LPR & 41.2 & 46.0 & 44.2 & 45.8 & 45.4 & 40.6 & 49.2 & 39.4 & 46.0 & 45.0 & 43.3 & 42.7 \\
\hline GdAB & 22.4 & 22.5 & 23.2 & 23.8 & 21.0 & 20.0 & 22.6 & 20.3 & 21.0 & 22.2 & 21.0 & 23.0 \\
\hline Gmb & 51.3 & 56.0 & 55.1 & 54.0 & 54.0 & 51.6 & 55.5 & 51.5 & 53.3 & 56.9 & 52.3 & 53.6 \\
\hline BdeAM & 48.0 & 50.6 & 50.3 & 50.3 & 49.8 & 48.6 & 52.3 & 48.7 & 47.9 & 52.7 & 51.2 & 51.6 \\
\hline GBOC & 27.4 & 29.9 & 28.7 & & 30.0 & 29.3 & 31.5 & 27.6 & 25.8 & 30.2 & & 31.7 \\
\hline GbbPp & 39.3 & 41.6 & 40.6 & & 39.6 & 39.0 & 42.5 & 37.5 & 39.0 & 43.2 & 38.3 & 43.0 \\
\hline GBFM & 15.8 & 17.0 & 16.0 & & 17.2 & 14.7 & 17.4 & 15.0 & 14.7 & 16.7 & 17.5 & 12.0 \\
\hline HFM & 13.2 & 12.7 & 14.0 & & 13.3 & 13.2 & 12.6 & 12.3 & 13.5 & 14.3 & 12.0 & 13.1 \\
\hline GNB & 51.3 & 53.0 & 53.0 & 54.5 & 53.5 & 49.7 & 53.0 & 52.0 & 50.6 & 52.0 & 53.3 & 54.7 \\
\hline ZB & 84.4 & 86.0 & & 86.6 & 86.4 & 80.3 & 92.0 & 86.0 & 87.5 & 89.8 & 85.5 & 88.5 \\
\hline LBS & 31.8 & 30.5 & 33.0 & 31.0 & 32.3 & 27.0 & 31.7 & 28.0 & 35.8 & 35.2 & 49.8 & 31.2 \\
\hline FB & 43.2 & 41.8 & 40.7 & 43.3 & 41.3 & 41.8 & 44.5 & 40.0 & 46.8 & 47.0 & 41.6 & 42.8 \\
\hline LbbO & 28.2 & 28.7 & 30.0 & 26.9 & 28.3 & 29.2 & 31.0 & 27.2 & 33.4 & 34.0 & 28.9 & 26.0 \\
\hline GPB & 47.4 & 48.7 & 51.0 & 51.3 & 49.8 & 49.5 & 53.5 & 51.7 & 50.3 & 50.4 & 49.7 & 52.0 \\
\hline LPB & 23.0 & 24.4 & 23.2 & 25.4 & 24.5 & 23.3 & 27.8 & 25.6 & 25.0 & 27.4 & 24.7 & 24.0 \\
\hline BCA & 25.9 & 26.3 & 24.8 & 27.4 & 27.0 & 25.7 & 30.5 & 27.7 & 27.4 & 27.0 & 25.7 & 26.8 \\
\hline GiHO & 28.0 & 28.7 & 28.3 & 29.0 & 28.0 & 27.0 & 28.3 & 26.3 & 27.0 & 28.0 & 29.4 & 29.0 \\
\hline SH & 44.9 & 48.0 & 47.3 & & 44.3 & 45.4 & 47.0 & 43.4 & 49.0 & 47.2 & 43.0 & 49.0 \\
\hline Hot & 36.4 & 41.0 & 38.5 & & 37.3 & 35.7 & 38.5 & 35.0 & 38.4 & 41.4 & 36.0 & 37.6 \\
\hline TIm & 110.6 & 118.9 & 115.0 & 118.6 & 118.5 & 113.0 & 125.7 & 110.8 & 116.8 & 122.2 & 112.6 & 123.3 \\
\hline Lapi & 112.5 & 120.0 & 115.5 & 120.7 & 120.0 & 114.2 & 126.8 & 110.8 & 117.0 & 120.6 & 113.4 & 124.7 \\
\hline Lii & 107.2 & 114.9 & 111.2 & 114.0 & 113.6 & 108.2 & 121.4 & 106.4 & 111.8 & 115.7 & 108.5 & 119.2 \\
\hline Lcpc & 99.0 & 103.7 & 102.0 & 115.5 & 105.0 & 99.4 & 111.2 & 98.0 & 104.2 & 109.5 & 98.0 & 109.6 \\
\hline Lic & 94.0 & 100.4 & 98.3 & 101.0 & 101.0 & 94.4 & 107.0 & 94.0 & 99.0 & 102.8 & 94.0 & 105.4 \\
\hline Lapc & 99.5 & 104.5 & 103.0 & 106.5 & 106.5 & 101.1 & 113.3 & 98.5 & 104.0 & 105.6 & 99.0 & 110.3 \\
\hline Lmr & 31.2 & 32.6 & 35.0 & 33.3 & 33.0 & 30.3 & 35.0 & 36.0 & 31.6 & 34.7 & 33.6 & 33.8 \\
\hline L C-M3 & 67.0 & 71.6 & 72.0 & 72.8 & 73.2 & 66.0 & 76.0 & 67.3 & 69.7 & 73.9 & 70.0 & 75.3 \\
\hline L P1-M3 & 62.0 & 68.0 & 68.2 & 67.0 & 66.6 & & 72.0 & 63.0 & 64.6 & 68.2 & 65.0 & 69.7 \\
\hline L P1-M2 & 58.6 & 64.0 & 63.7 & 62.8 & 63.2 & & 68.5 & 58.9 & 61.4 & 63.6 & 61.4 & 66.5 \\
\hline L P2-M3 & 57.5 & 62.7 & 63.5 & 61.2 & 61.4 & & 68.0 & 58.0 & 59.8 & 64.6 & 61.2 & 65.7 \\
\hline L P1-P4 & 32.5 & 36.4 & 34.0 & 34.0 & 35.3 & & 38.2 & 30.3 & 34.0 & 35.2 & 32.2 & 36.4 \\
\hline L P2-P4 & 27.7 & 31.0 & 29.1 & 28.6 & 29.4 & & 34.0 & 27.0 & 29.5 & 30.6 & 28.0 & 32.3 \\
\hline La M1 & 18.0 & 19.2 & 20.2 & 19.5 & 20.0 & 18.0 & 20.4 & 21.2 & 19.0 & 19.5 & 18.7 & 18.7 \\
\hline Hm P1 & 13.2 & 14.0 & 14.2 & 14.0 & 14.7 & 12.7 & 17.6 & 16.1 & 16.8 & 14.0 & 14.0 & 17.0 \\
\hline Hm P2P3 & 12.0 & 14.6 & 13.6 & 14.0 & 14.2 & 12.8 & 16.3 & 15.0 & 16.4 & 14.8 & 14.0 & 15.3 \\
\hline Hm M1 & 16.3 & 16.2 & 14.7 & 16.0 & 17.2 & 15.0 & 19.3 & 15.4 & 18.1 & 19.4 & 16.0 & 16.3 \\
\hline GT M1 & 7.0 & 6.8 & 7.2 & 8.1 & 8.2 & 7.5 & 9.1 & 8.6 & 8.0 & 7.2 & 7.9 & 7.5 \\
\hline HVR & 44.0 & 47.8 & 43.3 & 45.0 & 45.0 & 40.4 & 47.4 & 43.4 & 45.0 & 45.5 & 43.0 & 47 \\
\hline
\end{tabular}


Tab. 3 - Measures of the teeth of C. lupaster.

\begin{tabular}{|c|c|c|c|c|c|c|c|c|c|c|c|c|c|c|c|c|c|c|c|c|c|c|}
\hline \multirow{2}{*}{$\begin{array}{r}\mathbf{N} \\
\text { Side }\end{array}$} & \multicolumn{2}{|c|}{$\begin{array}{c}\text { MSNG } \\
26231\end{array}$} & \multicolumn{2}{|c|}{$\begin{array}{c}\text { MSNG } \\
26232\end{array}$} & \multicolumn{2}{|c|}{$\begin{array}{c}\text { MSNG } \\
26233\end{array}$} & \multicolumn{2}{|c|}{$\begin{array}{c}\text { MSNG } \\
31632\end{array}$} & \multicolumn{2}{|c|}{$\begin{array}{c}\text { MSNG } \\
31635\end{array}$} & \multicolumn{2}{|c|}{$\begin{array}{c}\text { MSNG } \\
34260\end{array}$} & \multicolumn{2}{|c|}{$\begin{array}{c}\text { MSNG } \\
26229\end{array}$} & \multicolumn{2}{|c|}{$\begin{array}{c}\text { MSNG } \\
26230\end{array}$} & \multicolumn{2}{|c|}{$\begin{array}{c}\text { MSNG } \\
26449\end{array}$} & \multicolumn{2}{|c|}{$\begin{array}{c}\text { MSNG } \\
31630\end{array}$} & \multicolumn{2}{|c|}{$\begin{array}{c}\text { MSNG } \\
32184 \\
\end{array}$} \\
\hline & $\mathrm{R}$ & L & $R$ & $\mathrm{~L}$ & $\mathrm{R}$ & $\mathrm{L}$ & $\mathrm{R}$ & L & $\mathrm{R}$ & L & $R$ & $\mathrm{~L}$ & $R$ & $\mathrm{~L}$ & $R$ & L & $R$ & L & $R$ & $\mathrm{~L}$ & $\mathrm{R}$ & $\mathrm{L}$ \\
\hline C L & & & & & & & & & .7 & & 6 & 6 & .0 & 0 & 0 & 1 & .3 & & .3 & 1 & .7 & 8.6 \\
\hline B & & 7 & 4.5 & & 7 & & & & 48 & & 0 & 9 & 5.0 & 3 & .6 & 7 & 5.0 & & .8 & 9 & .0 & 5.1 \\
\hline L & 7 & 4.7 & 5.5 & .5 & 4.8 & 0 & 2 & 2 & & & 8 & .7 & .0 & 2 & .0 & 8 & & & 5.4 & 4 & 5.2 & 5.0 \\
\hline $1 \mathrm{~B}$ & & 3.3 & 3.4 & 3.4 & 3.3 & 3.4 & 4 & 4 & & & 3 & 2 & 3 & .4 & .4 & .4 & & & 3.7 & 8 & 3.8 & 3.7 \\
\hline P2 L & & 8.2 & 5 & 9.6 & 9.3 & 9.1 & 9.0 & & & & 6 & & & 4 & .7 & .3 & .5 & 3 & 9.1 & 4 & 3 & 9.5 \\
\hline 2 B & & 3.5 & & 3.9 & 3.6 & 3.4 & 3.8 & & & & 6 & & 4.3 & 3 & 4.0 & 9 & .7 & 8 & 4.2 & .0 & 11 & 7.0 \\
\hline L & & 10.0 & 10.8 & 11.0 & 8 & 11.0 & \begin{tabular}{|l|l|}
10.7 \\
\end{tabular} & & & & 9.7 & 9.8 & 10.5 & 10.8 & 10.0 & 10.0 & & & \begin{tabular}{|l|}
10.7 \\
\end{tabular} & 10.5 & 11.3 & 11.4 \\
\hline B & & 4.0 & 1 & & 4.0 & & 4.6 & U & & 7 & 4.0 & 4.0 & 5.0 & 4.7 & 4.3 & 4.3 & & & \begin{tabular}{ll|}
4.3 \\
\end{tabular} & & 4.7 & 4.7 \\
\hline L & 6 & 6.7 & 3 & 18.3 & 7.7 & .0 & .2 & 3.3 & 18.5 & 8.3 & 15.6 & 15.7 & 18.3 & 8.3 & 17.0 & 17.0 & 18.0 & 18.7 & $\mid$\begin{tabular}{|l|} 
\\
\end{tabular} & 7.7 & 18.0 & 18.0 \\
\hline $1 \mathrm{E}$ & & & 0 & & 6.8 & & 7. & & 7.4 & & 6.3 & & 7.4 & & 6.6 & & 6.4 & & 7.0 & & 7.4 & 7.3 \\
\hline $3 \mathbf{P}$ & & & 3.6 & & 9.0 & & & & 8.8 & .0 & 8.0 & .7 & 10.0 & 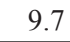 & 9.0 & & 8.6 & & 9.0 & .0 & 9.0 & 8.8 \\
\hline $11 \mathrm{~L}$ & 7 & .6 & 4 & .2 & 2 & .5 & & & 12.3 & 2.3 & 12.0 & 2.0 & 14.0 & 3.8 & 11.6 & 1.5 & 12.7 & 2.7 & \begin{tabular}{|l|}
12.0 \\
\end{tabular} & 2.3 & 2.3 & 12.3 \\
\hline I1 B & 2 & 13.5 & 14.0 & 14.6 & 14.3 & 14.7 & & .6 & 14.0 & 4.2 & 13.2 & 12.8 & 15.0 & 5.4 & 14.5 & 4.6 & 15.7 & 15.7 & \begin{tabular}{|l|}
15.2 \\
\end{tabular} & 5.8 & 15.2 & 15.7 \\
\hline M2 L & & 7.2 & & 7.6 & 7.5 & & 7.5 & & 7.5 & 7.4 & 7.2 & 7.0 & 8.2 & 8.0 & 7.0 & 6.7 & 7.6 & 0 & 7.7 & .0 & 8.3 & 8.0 \\
\hline M2 B & & 10.4 & 11.0 & 12.0 & 10.7 & & 11.0 & & 10.3 & 11.0 & 9.4 & 9.4 & 12.0 & 11.7 & 11.0 & 11.2 & 12.4 & 13.0 & \begin{tabular}{|l|}
11.0 \\
\end{tabular} & 1.5 & 11.6 & 11.4 \\
\hline C L & & 7.1 & 4 & & 7.4 & 7.3 & 7.6 & 7.8 & 8.2 & 8.4 & 7.4 & 7.6 & 5 & 8.6 & 8.0 & 8.0 & .0 & & 8.0 & & 3.0 & 8.3 \\
\hline & & & 5. & & 5 & & & & 5.2 & & 4.7 & 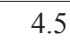 & 6. & & 6. & & 5.6 & & 5.4 & & 5.7 & \begin{tabular}{|l|}
5.6 \\
\end{tabular} \\
\hline $1 \mathrm{~L}$ & & 4 & 3 & & & & & & & & & & 3. & & 4.0 & & 3.6 & & 3.7 & & 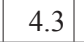 & 4.2 \\
\hline & & 2. & & & & & & & & & & & 3. & .0 & 3.0 & 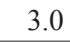 & 2.8 & & 3.2 & & .2 & 3.1 \\
\hline $\mathbf{L}$ & & & & & & & & & 8.7 & & & & & & .1 & & 8.3 & & 8.4 & & 8.1 & 8.3 \\
\hline & & & & & S & & & 0 & 4.4 & & & & & & & & 4.0 & 7.0 & 4.2 & 2 & 4.0 & 4.1 \\
\hline $3 \mathrm{~L}$ & & 8.0 & 0 & & 9.2 & 9.2 & 10.0 & .8 & 10.0 & & 8.7 & & & .5 & 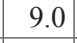 & 0 & .5 & .5 & 9.7 & 6 & 10.0 & 9.7 \\
\hline P3 B & & 8 & & 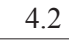 & 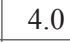 & 4.0 & 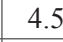 & 5 & 4.6 & & 4.2 & .0 & 3 & .3 & .3 & 1.5 & 4.3 & 4.2 & 4.2 & 2 & 4.4 & 4.4 \\
\hline P4 L & & 10.0 & 11.3 & 11.5 & 11.3 & 11.6 & 11.0 & 11.1 & 11.5 & 11.7 & 10.2 & 10.0 & 11.4 & 11.0 & 10.8 & 11.0 & 11.8 & 11.8 & \begin{tabular}{|l|} 
\\
\end{tabular} & 10.4 & 11.3 & 11.3 \\
\hline & & 4 & 5.0 & & 50 & & 56 & & 5.6 & 5 & 4.8 & 4.8 & 5.4 & 5.3 & 5.0 & 5.3 & 5.2 & 5.3 & 5.0 & 1 & 5.2 & 5.0 \\
\hline & & 18.3 & 19.6 & 19.7 & 20.5 & 20.4 & 20.0 & 20.0 & 20.7 & 20.7 & 18.0 & 18.0 & 22.1 & 21.6 & 19.2 & 19.4 & 20.6 & 20.0 & 20.5 & 20.3 & 20.0 & 19.3 \\
\hline & 7 & 7 & 7 & & 10 & & & & 8. & 8. & 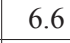 & & 0 & & 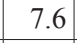 & 4 & 4 & 7. & 1.1 & & 7.7 & 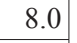 \\
\hline $\operatorname{Tr}$ & 6 & & & & 6.4 & & & & 6. & 0.0 & 6 & 5.0 & 6.3 & 6.6 & 6.1 & 3 & 7.2 & O & 6.5 & 4 & 0.1 & 6.3 \\
\hline M2 & & 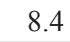 & 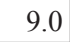 & & 10.2 & & & & 8. & & 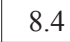 & 8.4 & 10.4 & 10.0 & 8.1 & 7 & 10.0 & 10.2 & \begin{tabular}{|l|} 
\\
\end{tabular} & 8 & 10.5 & 10.0 \\
\hline M2 B & 6. & 6.6 & . & & 6.7 & & 6.7 & & 7.0 & 7.0 & 5 & & 7.0 & 8 & .0 & 6.8 & 6.8 & 7.0 & 6.0 & 6 & 7.0 & $\begin{array}{ll}6.6 \\
\end{array}$ \\
\hline M3 L & 4. & 4.4 & 4. & 4 & & 5 & & & & & 4.0 & & 4.2 & & & & & & & & 4.8 & 4.7 \\
\hline M3 B & 4.0 & 4.0 & 4.0 & 4.2 & & 4.6 & & 4.3 & & & 3.6 & 3.6 & 5.0 & 4.7 & & & & & & & 4.5 & 4.6 \\
\hline
\end{tabular}

A series of box plots with selected craniodental measurements and the derived ratios were generated, thus providing a visual representation of how much C. lupaster differs from the other taxa for some key morphological features.

\section{RESULTS}

Morphological description

Considering the mean values, as previously noted by Ferguson (1981), C. lupaster is smaller than C. lupus but bigger than $C$. anthus. Body size is not the only difference, as morphological differences on skull and teeth can also be observed.

The shape of the nasal bones is reported in literature as a diagnostic character to separate wolf from golden jackal (Boitani, 2003); the nasal bones of C. lupaster are graph shaped as in Eurasian C. aureus and in C. anthus, while in $C$. lupus are V shaped. In C. lupaster the nasal bones are as long as the maxillary bones while in $C$. anthus they are shorter and in C. lupus they are longer. The forehead is low, and the angle between nasals and frontals is flat, while it is more evident in C. aureus. The palate is shorter than in C. lupus and C. aureus, ending in proximity of the mesial border of $\mathrm{M}^{2}$. A marked restriction of the palate width between $\mathrm{P}^{3}$ and $\mathrm{P}^{2}$ is present in $C$. anthus but not in C. lupus and C. lupaster. The sagittal crest is as low as in C. aureus, but this character could be due to an allometric development in small sized canids (Sardella et al., 2014). The pterigoid-palatine crest in distal portion is narrower than in mesial portion.

In C. lupus and C. aureus $\mathrm{P}^{2}$ and $\mathrm{P}^{3}$ have a secondary cusp and the distal cingulum is modified in an accessory cusp while in $C$. lupaster the accessory cusp is absent.

The upper carnassial is less thick than in C. lupus (Fig. 2). The paracone of $\mathrm{M}^{1}$ of $C$. lupaster is bigger than the metacone, as in C. lupus, while in C. aureus they are equal. In $C$. lupaster the protocone and the metaconule of $\mathrm{M}^{1}$ are much developed. $\mathrm{M}^{1}$ of $C$. lupaster has the protocone 


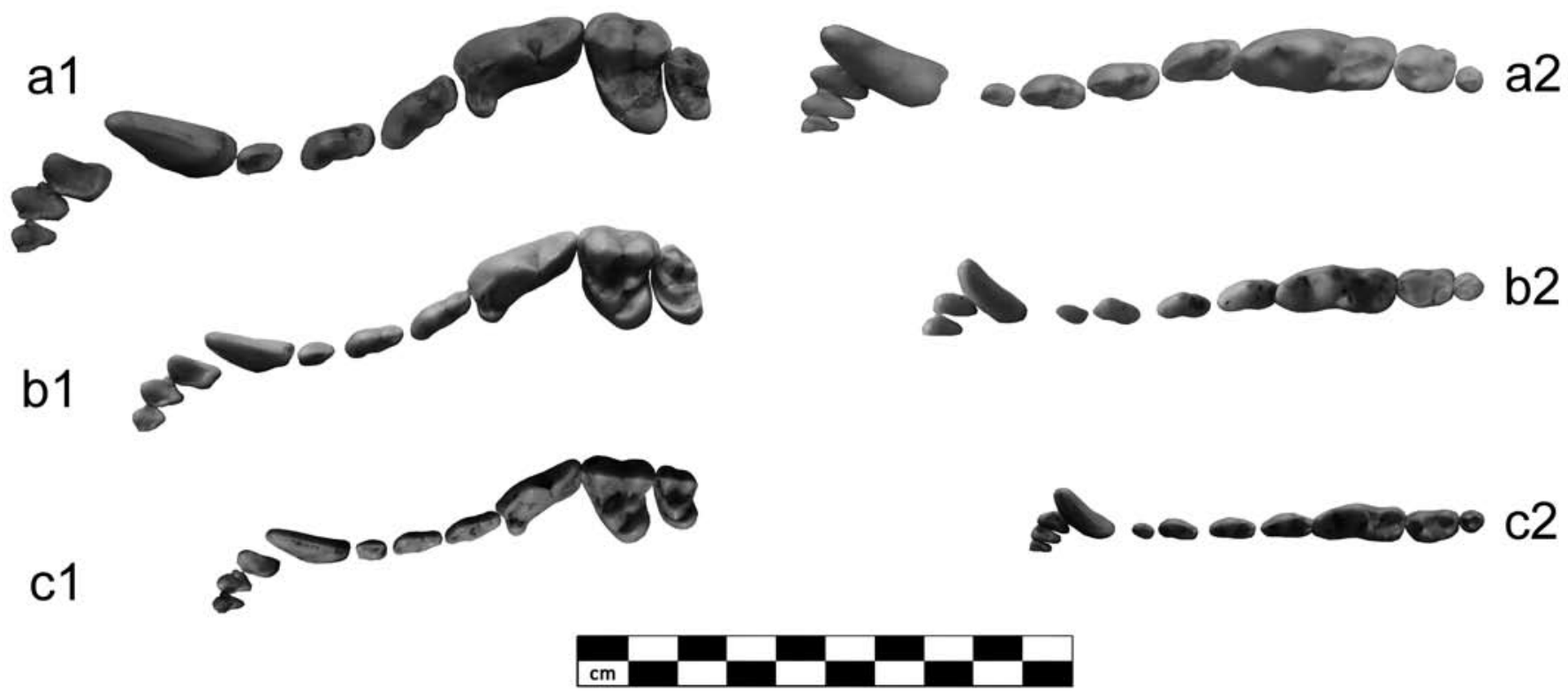

Fig. 2 - Teeth comparison; a1: upper teeth of C. lupus; a2) lower teeth of $C$ lupus; b1) upper teeth of C. lupaster; b2) lower teeth of $C$ lupaster; c1) upper teeth of $C$. anthus; c2) lower teeth of $C$. anthus.

mesially located; the metaconulo is small; the ipocone is well developed and clearly delineated; the basin is absent; the paraconule is large; the parastyle is even marked. $\mathrm{M}^{2}$ of $C$. lupaster has a big protocone, while the metaconule is absent or vestigial.

The mandible of $C$. lupaster is intermediate between those of $C$. lupus and $C$. anthus. The teeth of $C$. lupaster are less wide than those of $C$. lupus but are more robust than that of $C$. aureus (Fig. 2).

The $\mathrm{P}_{2}$ has a secondary cusp and the distal cingulum modified in an accessory cusp; in C. lupus another cusp is present. In the $\mathrm{P}_{3}$ are present two secondary cusps well developed, as in $C^{3}$. lupus; the $\mathrm{P}_{3}$ of $C$. lupaster is less large than that of $C$. lupus, but it is larger than the $\mathrm{P}_{3}$ of C. anthus is.

The trigonid of $\mathrm{M}_{1}$ is quite narrow and the big metaconid results prominent; the hypoconid is thick; the entoconid is visible. The $\mathrm{M}_{2}$ of $C$. lupaster has four cusps, and is more similar at $C$. anthus than at $C$. lupus; in C. lupus the entoconid of $\mathrm{M}_{2}$ is often absent or very reduced, while $C$. anthus shows a little entoconid located on the ridge of the cingulum; in occlusal view is quadrangular shaped (Fig. 1f). $\mathrm{M}_{3}$ of $C$. lupaster has only a central cusp as C. lupus, while in $C$. anthus are often present two cusps.

\section{Dental disease}

The specimens stored at Natural History Museum "G. Doria" of Genoa show a relative high percentage of dental disease. Various degrees of dental wear can be observed: MSNG 26231 shows little wear of the carnassial teeth and of the molars, MSNG 26230 has advanced wear of the teeth, with loss of many cusps, MSNG 31632 shows a more advanced wear. The specimen MSNG 26228 (Fig. 3) show advanced degree of dental wear. A dental abscess on right $\mathrm{M}^{1}$ probably caused a parodontal disease (Stillou et al., 2010) and a oronasal fistula (DuPont \& DeBowes, 2009).

\section{Morphometric description}

The MANOVA analysis performed on skull and mandible shows that the differences between mean are significant (Wilk's lambda: 0.0002138; P: 9.553E-159). The confusion matrix shows that all predicted groups are coincident with given groups and the specimens corrected classified are $100 \%$. A discriminant analysis, performed to test the differences between $C$. lupaster and $C$. anthus and $C$. lupus, is significant $(\mathrm{P}=1.68 \mathrm{E}-43)$. The PCA performed on skull and mandible shows similar differences (Fig. 4a). The species considered are well separated and distinct. The first component PC1 (vertical axis) accounts for $86.1 \%$ of the total variance, and has is loadings for TL. The second component (on the horizontal axis) explains $4.5 \%$ and is mainly influenced by LhP. ANOVA analysis on PC1 and PC2 has significant result (Tukey's pairwise test $\mathrm{P}=8.761 \mathrm{E}-06$ ).

The MANOVA analysis performed on upper and lower teeth shows that the differences between mean are significant (Wilk's lambda: 0.05184; P: 4.388E-136). The confusion matrix shows that just a specimen is classified as $C$. lupaster instead of $C$. anthus and viceversa. No errors of attribution are made with $C$. lupus. The OA value is 90.7. The three groups are well separated (Fig. 4b). A discriminant analysis, performed to test the differences between $C$. lupaster and $C$. anthus and $C$. lupus, is significant $(\mathrm{P}=1.68 \mathrm{E}-43)$. Another PCA is performed on upper and lower teeth: PC1 explains the $84.4 \%$ of the total variance and is mainly influenced by the length of the lower $\mathrm{M}_{1}$; PC2 explain the $2.68 \%$ of the variance and depends on the length of the upper canine. Considering only teeth $C$. lupaster and $C$. anthus are quite similar. ANOVA performed on PC1 and PC2 has significant result (Tukey's pairwise test $\mathrm{P}=8.761 \mathrm{E}-06$ ).

No sexual dimorphism is found considering carnassial teeth size, a common body size estimator (Van 
Valkenburgh, 1990). Sexual dimorphism is noticeable measuring the total length of the skull (TL). While C. anthus does not show sexual dimorphism for this character, C. lupus shows a similar degree of differentiation (Fig. 5a). The ratio LPR/LMR allows to clearly separate $C$. anthus from $C$. lupus and $C$. lupaster (Fig.5b). This difference is probably due to the diet of these animals, i.e. from the carnivorous diet of wolf to the more omnivorous diet of the golden jackal. Considering the palate, the ratio $\mathrm{GPB} / \mathrm{BCA}$ allows to separate $C$. lupaster from the others species considered (Fig. 5c). The palatal shape of C. lupaster shows difference in proportion. Considering the skull length, for example the ratio TL/CL (Fig. 5d), a little difference is observable, due to the difference in the position of the condyles.

\section{DISCUSSION AND CONCLUSIONS}

The taxonomic position of the golden jackal in Africa is more complex than previously supposed. Since 2011 genetic researches had suggested that some taxonomic classifications must be reconsidered (Rueness et al., 2011). Furthermore the African golden jackal has enough genetic differences to be considered apart from Eurasian golden jackal: following Koepfli et al. (2015) the Eurasian populations belong to $C$. aureus and the African populations belong to $C$. anthus. Some authors, using genetic data, recognise two lineages: $C$. lupaster and the "African golden jackal" (Gaubert et al., 2012). Also morphological differences were observed in the past between $C$. lupaster and the "African golden jackal", but not recognised as taxonomic level (Van Valkenburgh \& Wayne, 1994). This work propose the first morphological analysis on this specific

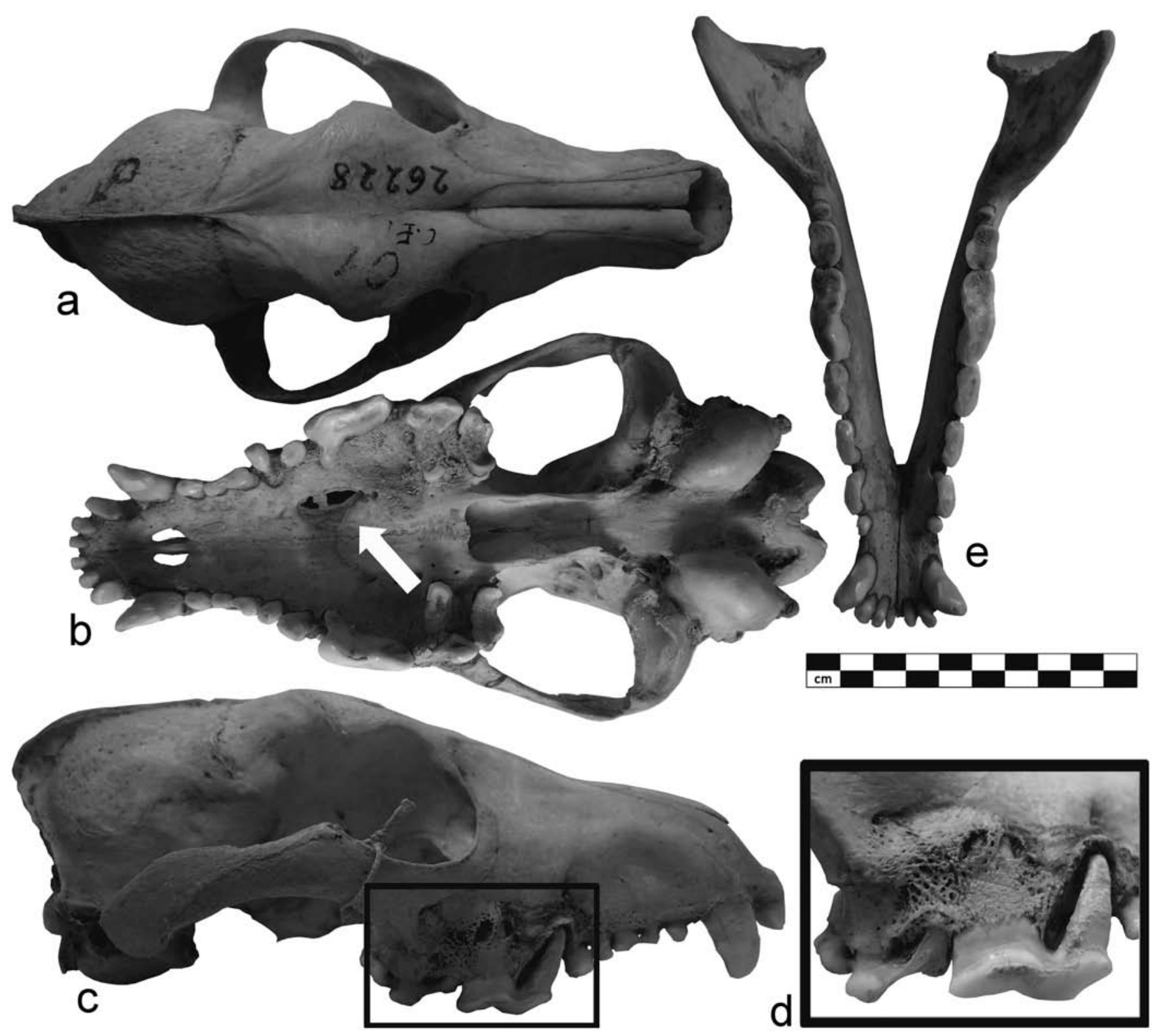

Fig. 3 - Specimen MSNG 26228; a) skull in dorsal view; b) skull in ventral view, oronasal fistula indicated from white arrow; c) skull in right lateral view; d) detail of the right upper carnassial; e) mandible in occlusal view. 
topic before the paper of Ferguson (1981). The specimens stored at Natural History Museum "G. Doria" of Genoa, despite the small sample size, show a coherent and homogeneous set of characters that suggest the possibility to identify some features to separate the two species. The present analysis show that $C$. lupaster and $C$. anthus differ in many ratios and proportions and they don't differ only in body-size.

Morphometric analysis applied in this study confirms the results of the genetic data. C. lupaster has some characters in common with $C$. lupus and others with $C$. an- thus but a multivariate analysis reveals that is well separate from the other species considered. Some of the features that remember $C$. anthus, such as the lack of a developed sagittal crest, are due to scale factors and must be considered as convergence. Future analyses on a bigger sample will be necessary to observe the variability degree of these characters in a population.

The Italian museums are full of specimens, most of them collected during colonial period, which can be very useful to answer modern question. The discussion about the taxonomic status of $C$. lupaster in the last years is
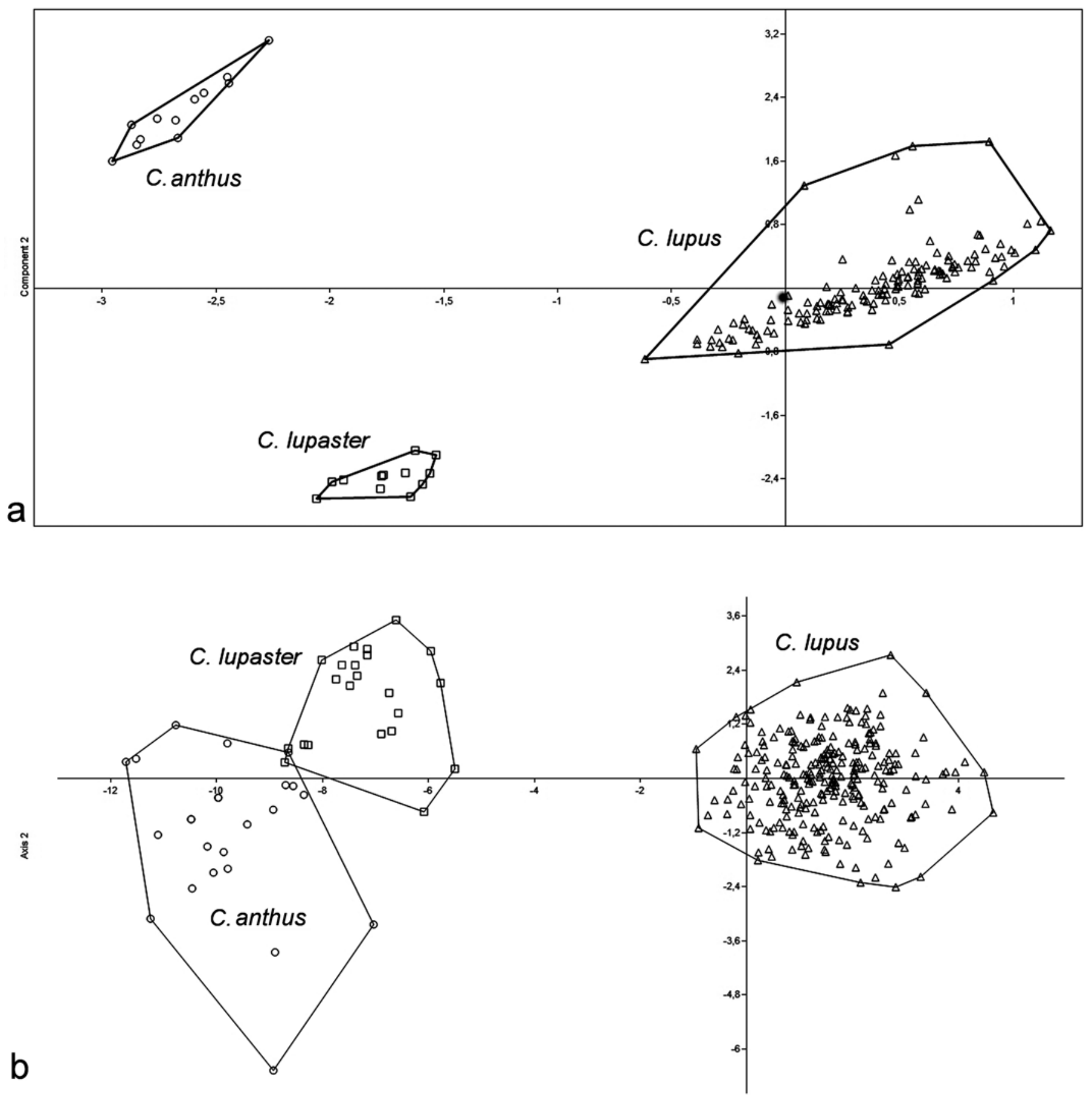

Fig. 4 - Multivariate analysis; triangle) C. lupus; square) C. lupaster; circle) C. anthus; a) PCA on skull and mandible; b) MANOVA on upper and lower teeth. 
remarkable. Many studies suggest that the biodiversity of African canids is probably more rich than previously supposed. A correct taxonomy is important to plan conservation actions and help threatened species and this paper would be a little help in order to improve our knowledge.

\section{Acknowledgements}

G. Doria (MSNG), A. de Faveri (ISPRA), G. Bardelli (MSNM), L. Boitani and A. Vigna Taglianti (DZR), M. Pavia (MRSNT), R. Sardella (Sapienza, University of Rome), C. Berto (University of Ferrara), A. Virtuoso (University of Copenhagen), D. Magnani (Istituto Zooprofilattico Sperimentale, Teramo), the anonymous reviewer.

\section{REFERENCES}

Anderson J. \& Winton W.E., 1902 - Zoology of Egypt, Mammalia. Hugh Rees Ltd, London.
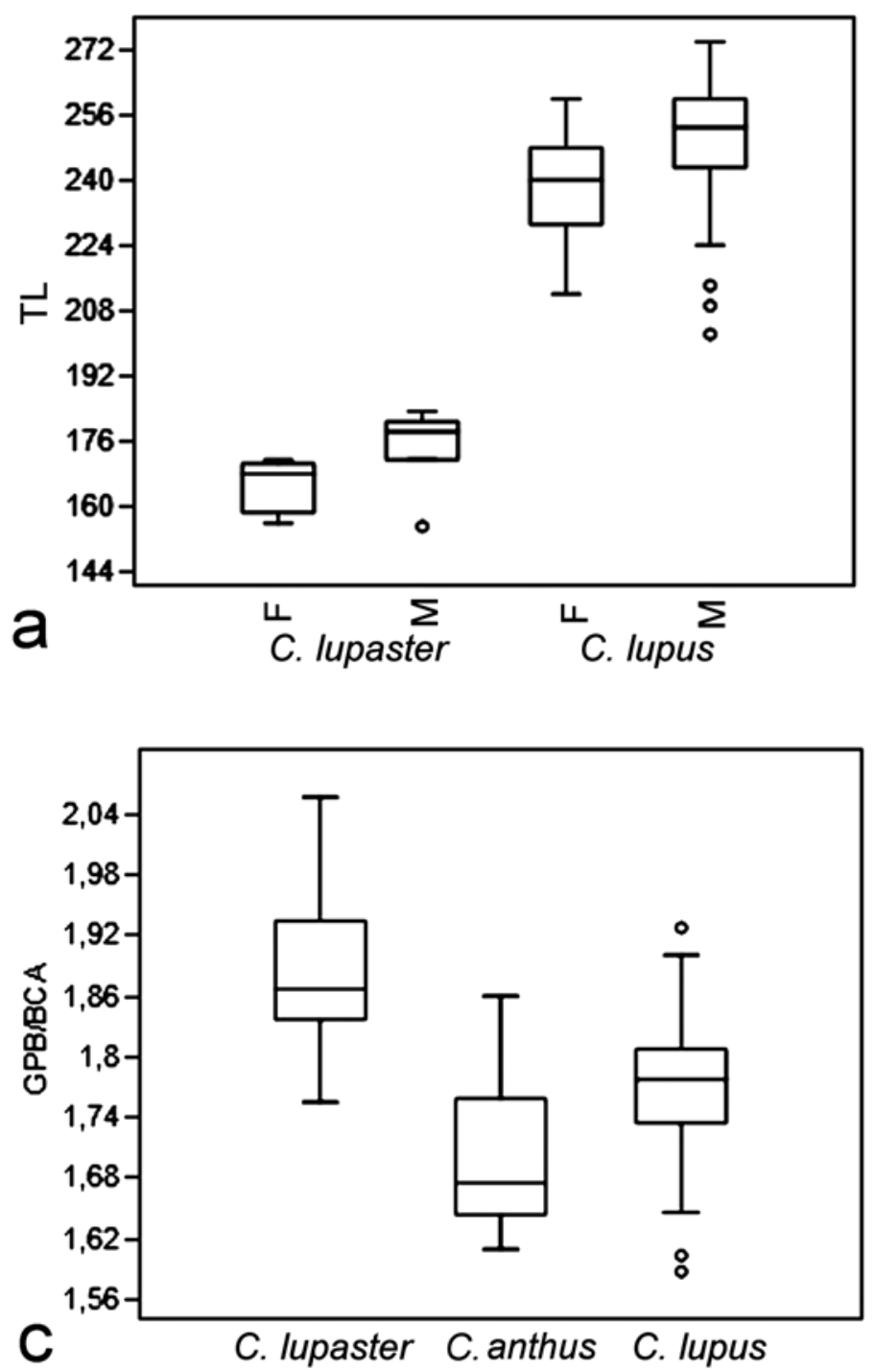

Beaux O., 1927 - Studien über neugeborene Säugetiere (äussere Form), Carnivora fissipedia. Zoologische Jahrbücher Abteilung für Systematik, 54: 1-38.

Bertè D.F., 2013 - L'evoluzione del genere Canis (Carnivora, Canidae, Caninae) in Italia dal wolf-event a oggi: implicazioni biocronologiche, paleoecologiche e paleoambientali. PhD thesis, Dipartimento di Scienze della Terra, Sapienza Università di Roma, Italia.

Boitani L., Lovari S. \& Vigna Taglianti A., 2003 - Fauna d'Italia. Mammalia III. Carnivora: Artiodactyla. Edizioni Calderini, Bologna.

DuPont G. \& DeBowes L., 2009 - Atlas of Dental Radiography in Dogs and Cats: A Practical Guide to Techniques and Interpretation. Saunders Elsevier Publications, Philadelphia.

Ellerman J.R. \& Morrison-Scott T.C.S., 1951 - Checklist of Palaearctic and Indian Mammals, 1758-1946. Trustees of the British Mus. Pub., London.
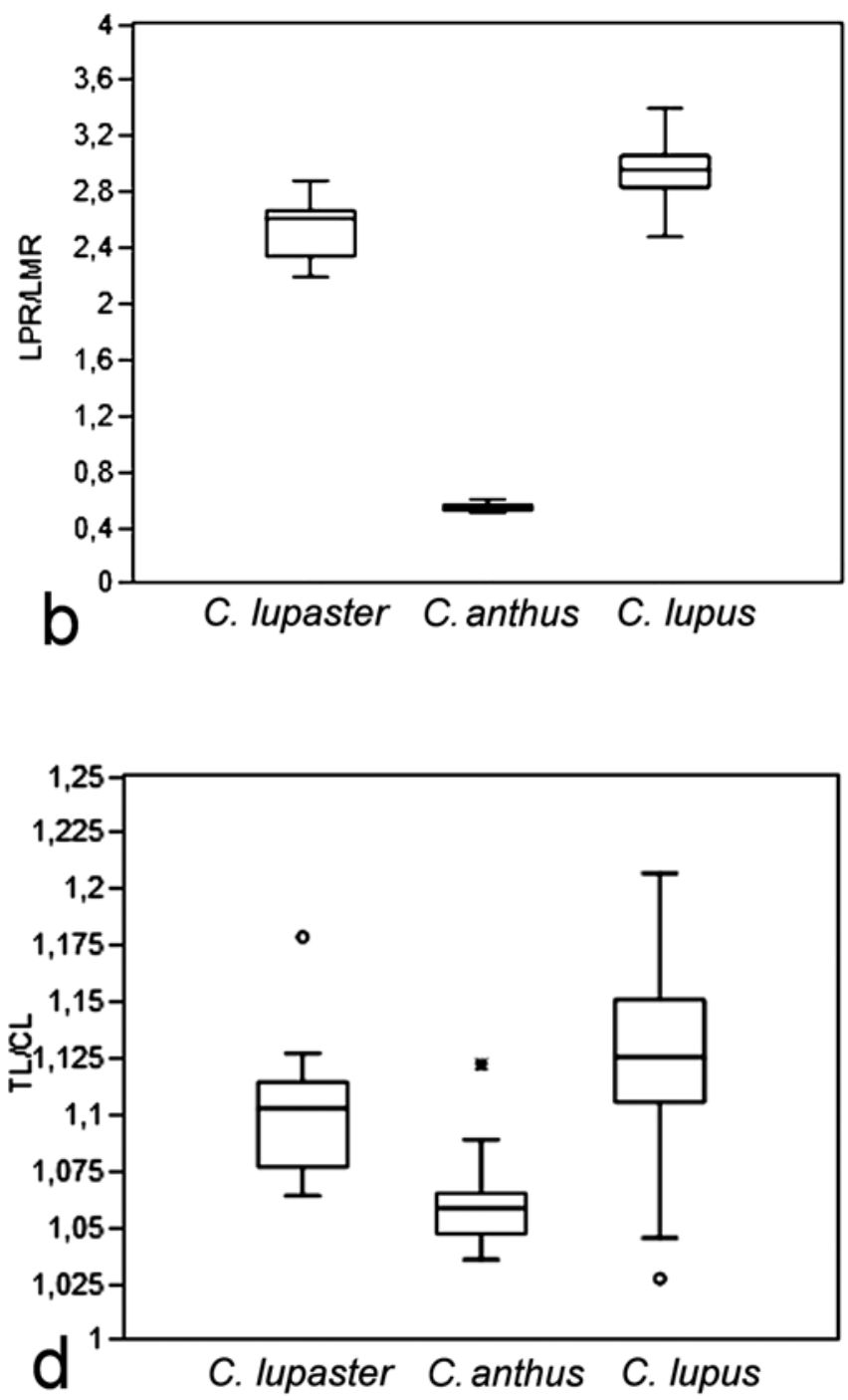

Fig. 5 - Box plots of selected absolute measurements and ratios; a) comparison of total length (TL) of C. lupaster and C. lupus male and female; b) ratio between length of premolars an length of molars (LPR/LMR); c) ratio between the great palatal breadth and the breadth measured at canine alveoli (GPB/BCA; d) ratio between total length and condilobasal length (TL/CL). 
Ferguson G.G., 1981 - The systematic position of Canis aureus lupaster (Carnivora: Canidae) and the occurrence of Canis lupus in North Africa, Egypt and Sinai. Mammalia, 45 (4): 459-466.

Flower S.S., 1932 - Notes on the recent mammals of Egypt, with a list of the species recorded from that kingdom. Proceedings of the Zoological Society of London: 369-450.

Gaubert P., Bloch C., Benyacoub S., Abdelhamid A., Pagani P., Adéyèmi C., Djagoun M.S., Couloux A. \& Dufour S., 2012 - Reviving the African Wolf Canis lupus lupaster in North and West Africa: a mitochondrial lineage ranging more than $6,000 \mathrm{~km}$ wide. $P L o S$ ONE, 7 (8): e42740.

Hammer Ø., Harper D.A.T. \& Ryan P.D., 2001 - PAST: Paleontological Statistics software package for education and data analysis. Paleontology electronica, 4 (1): 9.

Hoogstraal H., 1964 - A brief review of the contemporary land mammals of Egypt (including Sinai). 3. Carnivora, Hyracoidea, Perissodactyla and Artiodactyla. Journal of the Egyptian Public Health Association, 38: 205-239.

Hufnagl E., 1972 - Libyan Mammals. The Oleander Press, Cambridge.

Koepfli K.P., Pollinger J., Godinho R., Robinson J., Lea S., Hendricks S., Schweizer R.M., Thalmann O., Silva P., Fan Z., Yurchenko A.A., Dobrynin P., Makunin A., Cahill J.A., Shapiro B., Álvares F., Brito J.C., Geffen E., Leonard J.A., Helgen K.M., Johnson W.E., O'Brien S.J., Van Valkenburgh B. \& Wayne R.K., 2015 - Genome-wide evidence reveals that African and Eurasian golden jackals are distinct species. Current Biology, 25: 2158-2165.

Nowak R.M. \& Federoff N.E., 2002 - The systematic status of the Italian wolf Canis lupus. Acta Theriologica, 43: 333-338.

Rueness E.K., Asmyhr M.G., Sillero-Zubiri C., Macdonald D.W., Bekele A., Atikem A. \& Stenseth N.C., 2011 The Cryptic African Wolf: Canis aureus lupaster Is Not a Golden Jackal and Is Not Endemic to Egypt. PLOS ONE 6 (1): e16385.

Rueness E.K., Trosvik P., Atickem A., Sillero-Zubiri C., \& Trucchi E., 2015 - The African wolf is a missing link in the wolf-like canid phylogeny. bioRxiv, doi: https://doi.org/10.1101/017996

Sardella R., Bertè D.F., Iurino D.A., Cherin M. \& Tagliacozzo A., 2014 - The wolf from Grotta Romanelli (Apulia, Italy) and its implications in the evolutionary history of Canis lupus in the Late Pleistocene of Southern Italy. Quaternary International, 328-329: 179-195.

Setzer H.W., 1961 - The canids (Mammalia) of Egypt. Journal of the Egyptian Public Health Association, 36: 113-118.

Schwarz E., 1926a - Über Typenexemplare von Schakalen. Senckenbergiana, Frankfurt a.M., 8: 39-47.

Schwarz E., 1926b - Der Schakal der Galla-Hochlander. Variationsstudien an Säugetieren, I. Senckenbergiana, Frankfurt a.M., 8: 155-158.
Spassov N. \& Stoyanov S., 2014 - On the specific taxonomical status of the wolf-jackal Canis lupaster. Book of abstracts. First International Jackal Symposium. 1316 October 2014. Veliko Gradište, Serbia: 20.

Strillou X., Boutigny H., Soueidan A. \& Layrolle P., 2010 Experimental animal models in periodontology: a review. The Open Dentistry Journal, 4: 37-47.

Urios V., Donat-Torres M.P., Ramírez C., Monroy-Vilchis O. \& Rgribi-Idrissi H., 2015 - El análisis del genoma mitocondrial del cánido estudiado en Marruecos manifiesta que no es ni lobo (Canis lupus) ni chacal euroasiático (Canis aureus). AltoterO 3: 1-24.

Van Valkenburgh B., 1990 - Skeletal and dental predictors of body mass in carnivores. In: Body Size in Mammalian Paleobiology: Estimation and Biological Implications. Damuth J. \& MacFadden B.J. (eds.). Cambridge University Press, Cambridge: 181-205.

Van Valkenburgh B. \& Wayne R.K., 1994 - Shape divergence associated with size convergence in sympatric East African jackals. Ecology, 75: 1567-1581.

Von den Driesch A., 1976 - A guide to the measurement of animal bones from archaeological sites. Peabody Museum Bulletin, Harvard, 1.

Zammarano F.E., 1930 - Le Colonie italiane di diretto dominio. Fauna e caccia. Ministero delle Colonie, Roma. 